\title{
Variation in Use of High-Cost Technologies for Palliative Radiation Therapy by Radiation Oncologists
}

Aileen B. Chen, MD, MPP1; Jiangong Niu, $\mathrm{PhD}^{1}$; Angel M. Cronin, $\mathrm{MS}^{2}$; Ya-Chen Tina Shih, $\mathrm{PhD}^{1}$; Sharon Giordano, MD, $\mathrm{MPH}^{1}$; and Deborah Schrag, MD, $\mathrm{MPH}^{2}$

\begin{abstract}
Background: Understanding the sources of variation in the use of high-cost technologies is important for developing effective strategies to control costs of care. Palliative radiation therapy (RT) is a discretionary treatment and its use may vary based on patient and clinician factors. Methods: Using data from the SEER-Medicare linked database, we identified patients diagnosed with metastatic lung, prostate, breast, and colorectal cancers in 2010 through 2015 who received $\mathrm{RT}$, and the radiation oncologists who treated them. The costs of radiation services for each patient over a 90-day episode were calculated, and radiation oncologists were assigned to cost quintiles. The use of advanced technologies (eg, intensity-modulated radiation, stereotactic $\mathrm{RT}$ ) and the number of $\mathrm{RT}$ treatments (eg, any site, bone only) were identified. Multivariable random-effects models were constructed to estimate the proportion of variation in the use of advanced technologies and extended fractionation (>10 fractions) that could be explained by patient fixed effects versus physician random effects. Results: We identified 37,361 patients with metastatic lung cancer, 3,684 with metastatic breast cancer, 5,323 with metastatic prostate cancer, and 8,726 with metastatic colorectal cancer, with $34 \%, 27 \%, 22 \%$, and $9 \%$ receiving RT within the first year, respectively. The use of advanced technologies and extended fractionation was associated with higher costs of care. Compared with the patient case-mix, physician variation accounted for a larger proportion of the variation in the use of advanced technologies for palliative RT and the use of extended fractionation. Conclusions: Differences in radiation oncologists' practice and choices, rather than differences in patient case-mix, accounted for a greater proportion of the variation in the use of advanced technologies and high-cost radiation services.
\end{abstract}

J Natl Compr Canc Netw 2021;19(4):421-431 doi: 10.6004/jnccn.2020.7633

\footnotetext{
${ }^{1}$ The University of Texas MD Anderson Cancer Center, Houston, Texas; and ${ }^{2}$ Division of Population Sciences and the Center for Outcomes and Policy Research, Dana-Farber Cancer Institute, Boston, Massachusetts.
}

\section{Background}

Traditional fee-for-service payment models reward physicians for providing greater quantities of services and may have contributed to unsustainable increases in healthcare spending. This trend has spurred an increasing interest in alternative payment models, including recently proposed episode-based payment models for radiation oncology. ${ }^{1}$ Many payment models seek to make physicians accountable for the overall costs of the services that they provide, with the goal of reducing unnecessary and high-cost care. ${ }^{2}$ Critics, however, argue that much of the variation in the costs of care is a result of differences in the patient case-mix, ${ }^{3}$ which is difficult for providers to control and challenging for payment models to adequately account for, rather than provider behavior, and that alternative payment models may lead to incentives to reduce essential or discretionary care. Although quality measures can be used to counter incentives to reduce care, highly discretionary treatments such as palliative care can have outcomes such as pain control that are more difficult to measure and can thus be at greater risk of underuse.

More than one-third of patients with metastatic cancer will receive palliative radiation therapy (RT) during the course of their disease. The costs of palliative RT under a fee-for-service model are primarily driven by 2 factors: number of treatments received and use of higher-cost advanced technologies. Given the limited life expectancy of many patients with metastatic cancers and often-delayed clinical responses to palliative RT, most studies have advocated for shorter courses of treatment. ${ }^{4}$ In addition, aside from select patients with oligometastatic disease,,$^{5-8}$ there is little evidence to suggest that the use of advanced technologies, such as intensity-modulated RT (IMRT) and stereotactic body RT, or a greater number of treatments is beneficial in the palliative setting for most patients., ${ }^{4-11}$

See JNCCN.org for supplemental online content. 
To address the increasing costs of care, it is important to understand to what extent variation in the use of high-cost technologies and the number of treatments for palliative RT can be attributed to differences in patient and disease characteristics versus systematic differences in radiation oncologists' choices. This information can then be used to design payment models that promote the efficient use of resources in a manner that aligns care quality, value, and patient interests. If variation in care occurs predominantly because of clinical factors, then it could reflect appropriate decision-making by physicians treating patients with different characteristics. However, if this variation is largely explained by systematic differences in radiation oncologists' preferences for care, then payment models that seek to reduce variation in care may be justified. This study evaluated variation in the use of advanced radiation technologies and the number of radiation treatments received for patients with metastatic lung, breast, prostate, or colorectal cancer insured by Medicare fee-for-service plans by estimating the proportion of variation that could be accounted for by patient case-mix versus radiation oncologist choices.

\section{Methods}

\section{Cohort}

\section{Full Cohort}

We identified patients who were diagnosed with metastatic lung, breast, prostate, or colorectal cancer in 2010 through 2015 (AJCC 7th edition stage IV lung, breast, or colorectal cancer or stage IV prostate cancer with distant metastases) and who initiated RT within 1 year after diagnosis. To avoid radiation courses where treatment was planned but never delivered, we required all patients in the cohort to have at least 1 claim for radiation delivery.

\section{Bone Metastases Cohort}

In addition, from the cohort of patients receiving RT, we identified a secondary subgroup of patients who received RT for bone metastases. Radiation for bone metastases was defined if the patient had SEER-identified bone metastases (CS Mets at DX-Bone) at diagnosis and if the claims for their first course of radiation were associated with an ICD-9 diagnosis of bone metastases (ICD-9-CM diagnosis code 198.5).

\section{Radiation Episode}

RT typically consists of radiation planning followed by treatment delivery. We characterized an RT episode by first defining an index RT delivery date as the first date of service associated with an RT delivery code, using
Medicare claims. We then identified claims for RT planning occurring no more than 28 days before and no more than 3 days after the index RT delivery date. The RT episode start date was defined as the index RT delivery date or the associated RT planning date, whichever came first. The cohort assembly diagram is shown in supplemental eFigure 1 (available with this article at JNCCN.org). We considered an RT episode as lasting 90 days from the episode start date, based on the length of RT episodes currently under consideration in the Centers for Medicare \& Medicaid Services proposed alternative payment model. ${ }^{12,13}$ The $9.6 \%$ of patients who had claims consistent with a second course of RT, which we defined as a second RT simulation claim within the 90-day episode, were excluded. Sensitivity analyses were performed using a 30-day RT episode length and included patients treated using multiple courses of RT during the episode.

\section{Estimation of Medicare Spending}

We used Medicare claims to estimate total Medicare spending on radiation services during the 90-day RT episode. Claims files included hospital outpatient and physician/carrier files, which accounted for both hospital-based and freestanding radiation facilities. All spending estimates were from the perspective of the Medicare program and were based on the Medicare payment variables in the outpatient and physician/carrier claims files to include both professional and technical charges from RT. We used the medical care component of the Consumer Price Index to adjust for inflation, and all costs were calculated based on 2018 US dollars. ${ }^{14,15}$

\section{Outcomes}

For each patient, the type of RT delivered (external-beam RT [EBRT; includes non-IMRT EBRT and IMRT]) and stereotactic RT was determined based on the technical complexity of treatment delivery from claims within the first radiation course (stereotactic RT $>$ IMRT $>$ non-IMRT EBRT). For patients treated using EBRT, the number of fractions was defined by summing the number of unique dates of service associated with an RT delivery code within the 90-day episode.

We considered the following RT outcomes for the full cohort: (1) number of fractions of EBRT, categorized into $\leq 10$ versus $>10$ fractions (extended fractionation); (2) IMRT versus non-IMRT EBRT; and (3) stereotactic RT versus EBRT. For the bone metastases cohort, we considered the outcome of $>10$ versus $\leq 10$ fractions of EBRT for bone metastases.

\section{Physician Assignment}

One radiation oncologist was assigned to each patient, using encrypted National Provider Identifiers (NPIs) 
associated with RT claims. Assignment was prioritized as follows so that each patient was assigned to a single radiation oncologist: first to the physician associated with the first clinical RT planning (93\% of patients), followed by the physician associated with the patient's first treatment management claim ( $6 \%$ of patients), followed by the physician responsible for the patient's first RT claim of any type ( $1 \%$ of patients).

\section{Statistical Analysis}

For each outcome, the proportion of patients within each treatment/outcome group was summarized by means and percentages. We also summarized the distribution of Medicare payments by RT group and average RT costs per patient by physician. Considering that differences in Medicare spending on RT are driven primarily by variation in treatment practices, we constructed multivariable random-effects logistic regression models to examine the proportion of variation in treatment outcomes explained by components of the multilevel model: (1) patient-level fixed effects (age, sex, modified Charlson comorbidity index score, ${ }^{16-18}$ marital status, year of diagnosis, disease type, and presence of metastases in bone, brain, liver, and/or lung); and (2) physician-level random effects (random intercept).

For a subset of $72 \%$ of patients, we were able to obtain data on their radiation oncologist's sex, year of graduation, and graduation from either a US or non-US medical school using the American Medical Association (AMA) Physician Masterfile. A subgroup analysis was conducted that controlled for these physician characteristics as additional variables.

Following a latent variable approach (ie, assuming that the observed dichotomous treatment outcome is based on an underlying latent continuous propensity for receiving a particular treatment), total variation was defined by: ${\sigma_{\mathrm{F}}}^{2}+{\sigma_{\mathrm{R}}}^{2}+\pi^{2} / 3$, where $\sigma_{F}^{2}$ is the variance of the linear predictors from the fixed part of the model and $\sigma_{R}^{2}$ is the variance of the random effects.

The variation attributable to the fixed effects, represented by $\sigma_{\mathrm{F}}^{2} /\left(\sigma_{\mathrm{F}}^{2}+{\sigma_{\mathrm{R}}}^{2}+\pi^{2} / 3\right)$, was estimated by the sample variance of the fixed-effects linear predictor. The variation attributable to the physician random effects, represented by $\sigma_{\mathrm{R}}{ }^{2} /\left(\sigma_{\mathrm{F}}^{2}+\sigma_{\mathrm{R}}{ }^{2}+\pi^{2} / 3\right)$, was estimated by the random-effects variance. ${ }^{19}$

Because providers may choose treatments based on factors affecting prognosis that are not fully captured in the SEER-Medicare data, we performed additional analyses separating the cohort into patients who survived $<6$ versus $\geq 6$ months.

\section{Results}

Using data from the SEER-Medicare linked database, we identified patients diagnosed with metastatic lung

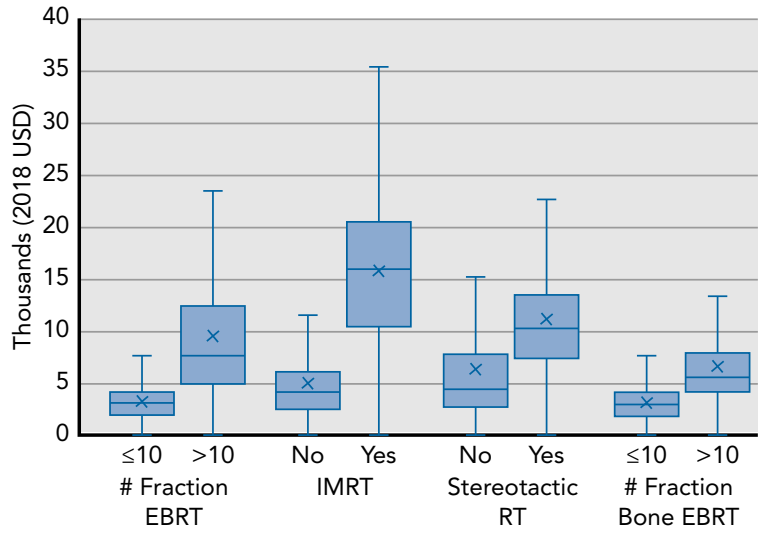

Figure 1. Medicare reimbursement for radiation therapy, by technology and number of fractions. Restricted to patients without multiple clinical treatment planning codes within the 90-day episode. Reimbursements for number of fractions, IMRT, and stereotactic RT did not control for site of treatment. Horizontal line: mean; X: median; thin bars: maximum/minimum; shaded box: interquartile range. Abbreviations: EBRT, external-beam radiation therapy; IMRT, intensitymodulated radiation therapy; RT, radiation therapy.

$(\mathrm{n}=37,361)$, prostate $(\mathrm{n}=5,323)$, breast $(\mathrm{n}=3,684)$, and colorectal $(n=8,726)$ cancers in 2010 through 2015 who satisfied the inclusion criteria. Among these patients, 12,626 (34\%) with lung cancer, 992 (27\%) with breast cancer, $1,174(22 \%)$ with prostate cancer, and $761(9 \%)$ with colorectal cancer received a course of EBRT or stereotactic RT within 1 year of diagnosis. Construction of the full study cohort is summarized in supplemental eFigure 1 . For the secondary subgroup, a total of 4,193 patients $(2,931$ with lung cancer, 465 with breast cancer, 707 with prostate cancer, and 90 with colorectal cancer) had bone metastases at diagnosis recorded in SEER and had a first course of RT that was associated with an ICD-9 diagnosis of bone metastases.

Patient and disease characteristics by disease site are summarized in Table 1 . Overall, $47 \%, 31 \%, 19 \%$, and $23 \%$ of patients had SEER-identified bone, brain, liver, and lung metastases at diagnosis, respectively. Distribution of metastatic sites differed by diagnosis. For example, $43 \%$ of patients with lung cancer, $73 \%$ with breast cancer, $90 \%$ with prostate cancer, and $15 \%$ with colorectal cancer had bone metastases at diagnosis. We identified 1,741 unique radiation oncologists in our cohort, with a median number of patients per physician of 5 (range, 1-64).

Figure 1 summarizes unadjusted Medicare reimbursements for palliative RT by fractionation and use of radiation technologies. In our cohort, $12 \%$ of patients treated using EBRT received IMRT, and 9\% treated using EBRT or stereotactic RT received stereotactic RT. Overall, $49 \%$ of patients treated at any 


\section{Table 1. Characteristics of Overall Cohort, by Cancer Type}

\begin{tabular}{|c|c|c|c|c|c|}
\hline & $\begin{array}{l}\text { Lung } \\
\text { n (\%) }\end{array}$ & $\begin{array}{c}\text { Breast } \\
\text { n (\%) }\end{array}$ & $\begin{array}{c}\text { Prostate } \\
\text { n (\%) }\end{array}$ & $\begin{array}{c}\text { Colorectal } \\
\text { n (\%) }\end{array}$ & $\begin{array}{c}\text { Overall } \\
\text { n (\%) }\end{array}$ \\
\hline Total patients, $\mathrm{N}$ & 12,626 & 992 & 1,174 & 761 & 15,553 \\
\hline \multicolumn{6}{|l|}{ Age group, y } \\
\hline $66-69$ & $3,253(26)$ & $255(26)$ & $241(21)$ & $188(25)$ & $3,937(25)$ \\
\hline $70-74$ & $3,799(30)$ & $250(25)$ & $302(26)$ & $193(25)$ & 4,544 (29) \\
\hline $75-79$ & $2,851(23)$ & $203(20)$ & $244(21)$ & $152(20)$ & $3,450(22)$ \\
\hline$\geq 80$ & $2,723(22)$ & $284(29)$ & 387 (33) & $228(30)$ & $3,622(23)$ \\
\hline \multicolumn{6}{|l|}{ Sex } \\
\hline Male & $6,612(52)$ & $15(2)$ & $1,174(100)$ & $390(51)$ & $8,191(53)$ \\
\hline Female & $6,014(48)$ & 977 (98) & $0(0)$ & 371 (49) & $7,362(47)$ \\
\hline \multicolumn{6}{|l|}{ Race } \\
\hline White & $10,974(87)$ & $849(86)$ & $996(85)$ & $646(85)$ & $13,465(87)$ \\
\hline Black & $1,029(8)$ & $109(11)$ & $118(10)$ & $75(10)$ & $1,331(9)$ \\
\hline Other & $623(5)$ & $34(3)$ & $60(5)$ & $40(5)$ & 757 (5) \\
\hline \multicolumn{6}{|l|}{ Rural residence } \\
\hline No & $10,220(81)$ & $833(84)$ & $958(82)$ & $623(82)$ & $12,634(81)$ \\
\hline Yes & 2,406 (19) & $159(16)$ & $216(18)$ & $138(18)$ & 2,919 (19) \\
\hline \multicolumn{6}{|c|}{ College education quintile ${ }^{a}$} \\
\hline 1 (lowest) & $2,663(21)$ & $161(16)$ & 218 (19) & $167(22)$ & $3,209(21)$ \\
\hline 2 & $2,598(21)$ & $183(18)$ & $223(19)$ & $151(20)$ & $3,155(20)$ \\
\hline 3 & $2,490(20)$ & $211(21)$ & $232(20)$ & $156(21)$ & $3,089(20)$ \\
\hline 4 & $2,503(20)$ & $234(24)$ & $233(20)$ & $138(18)$ & $3,108(20)$ \\
\hline 5 & 2,372 (19) & $203(20)$ & $268(23)$ & $149(20)$ & 2,992 (19) \\
\hline \multicolumn{6}{|l|}{ State Medicaid buy-in } \\
\hline No & $10,687(85)$ & $840(85)$ & $1,041(89)$ & $629(83)$ & $13,197(85)$ \\
\hline Yes & $1,939(15)$ & $152(15)$ & $133(11)$ & $132(17)$ & $2,356(15)$ \\
\hline \multicolumn{6}{|l|}{ Poverty rate } \\
\hline Low $(<10 \%)$ & $5,588(44)$ & $499(50)$ & $573(48)$ & $333(43)$ & $6,993(45)$ \\
\hline High ( $\geq 10 \%$ ) & $7,038(56)$ & $493(50)$ & $601(51)$ & $428(56)$ & $8,560(55)$ \\
\hline \multicolumn{6}{|l|}{ Marriage status } \\
\hline Married/Partner & $6,822(54)$ & $377(38)$ & $747(64)$ & $368(48)$ & $8,314(53)$ \\
\hline Single & $5,804(46)$ & $615(62)$ & $427(36)$ & $393(52)$ & $7,239(47)$ \\
\hline \multicolumn{6}{|l|}{ Modified $\mathrm{CCl}$ score ${ }^{\mathrm{b}}$} \\
\hline 0 & $6,134(49)$ & $635(64)$ & $712(61)$ & $490(64)$ & $7,971(51)$ \\
\hline 1 & $3,416(27)$ & $211(21)$ & $237(20)$ & $152(20)$ & $4,016(26)$ \\
\hline$\geq 2$ & $3,076(24)$ & $146(15)$ & 225 (19) & $119(16)$ & $3,566(23)$ \\
\hline \multicolumn{6}{|l|}{ Treatment location } \\
\hline Freestanding center & $3,960(31)$ & $305(31)$ & $452(39)$ & $228(30)$ & $4,945(32)$ \\
\hline Hospital & $8,666(69)$ & $687(69)$ & $722(62)$ & $533(70)$ & $10,608(68)$ \\
\hline \multicolumn{6}{|l|}{ Metastases at diagnosis ${ }^{c}$} \\
\hline Bone & $5,478(43)$ & $725(73)$ & $1,062(90)$ & $111(15)$ & $7,376(47)$ \\
\hline Brain & $4,561(36)$ & $118(12)$ & $18(2)$ & $50(7)$ & $4,747(31)$ \\
\hline Liver & $2,317(18)$ & $167(17)$ & $45(4)$ & $456(60)$ & 2,985 (19) \\
\hline Lung & $3,014(24)$ & $261(26)$ & $83(7)$ & 267 (35) & 3,625 (23) \\
\hline
\end{tabular}




\begin{tabular}{|c|c|c|c|c|c|}
\hline 2010 & 2,291 (18) & $168(17)$ & $162(14)$ & $156(21)$ & $2,777(18)$ \\
\hline 2011 & $2,096(17)$ & 177 (18) & $184(16)$ & $138(18)$ & 2,595 (17) \\
\hline 2014 & $2,017(16)$ & 177 (18) & $214(18)$ & 127 (17) & 2,535 \\
\hline 2015 & $1,978(16)$ & $152(15)$ & $234(20)$ & $116(15)$ & $2,480(16)$ \\
\hline \multicolumn{6}{|l|}{ Region } \\
\hline Northeast & $2,422(19)$ & $242(24)$ & $247(21)$ & $159(21)$ & $3,070(20)$ \\
\hline
\end{tabular}

Abbreviation: $\mathrm{CCl}$, Charlson comorbidity index.

apercent of persons aged $\geq 25$ years with at least 4 years of college education in the patient's census tract.

bDetermined from claims from 1 month through 13 months before diagnosis. Deyo-Romano-Klabunde modification of the Charlson score. ${ }^{16-18}$

'Not mutually exclusive. Based on SEER variables that identify sites of distant metastatic involvement at the time of diagnosis. Patients with unknown status for a particular site ( $2 \%$ for bone metastasis, $3 \%$ for brain metastasis, $3 \%$ for liver metastasis, and $4 \%$ for lung metastasis) were designated as not having involvement at that site.

site and $33 \%$ of patients treated for bone metastases received $>10$ fractions during a 90-day episode. Using a greater number of fractions or more advanced technologies was associated with higher costs. For example, the mean reimbursement for 1 to 10 fractions of radiation was $\$ 3,246$ compared with $\$ 9,578$ for $>10$ fractions. Likewise, non-IMRT versus IMRT episodes cost a mean of $\$ 5,057$ versus $\$ 15,813$, and nonstereotactic EBRT versus stereotactic RT episodes cost a mean of $\$ 6,331$ versus $\$ 11,122$.

When physicians were divided into quintiles by mean radiation cost per patient, we found that physicians in the highest cost quintiles were more likely to use a greater number of radiation treatments and were more likely to use stereotactic RT and IMRT treatments. For example, the mean number of radiation fractions used for bone metastases was 6.7 versus 16.4 for physicians in the lowest versus the highest cost quintiles. Furthermore, physicians in the lowest versus the highest cost quintiles used IMRT for $3 \%$ versus $45 \%$ of their patients insured by Medicare. Figure 2 shows mean Medicare reimbursement, use of stereotactic RT, use of IMRT, and radiation fractionation for the full cohort along with radiation fractionation for the bone metastases cohort by physician cost quintile. Sensitivity analysis that considered an episode to be 30 days revealed similar results, although the mean number of fractions was reduced compared with a 90-day episode.

Because variation in practice may result from differences in the patient case-mix, we sought to determine the proportion of the variation in use that was a consequence of differences in patient case-mix versus differences in physician practice patterns. Tables 2 and 3 provide the results of the multilevel models, which shows the effect of patient-case mix variables compared with physician effects on the variation in the use of radiation technologies and fractionation in the study cohort. We found that for the use of all advanced technologies and of extended fractionation, practice pattern variations among radiation oncologists accounted for a larger proportion of the observed variation than did patient case-mix. Physician effects accounted for $31.8 \%$ of the variation in IMRT use, whereas patient case-mix accounted for $16.9 \%$. Likewise, physician effects accounted for $43.2 \%$ of the variation in the use of stereotactic RT compared with $15.1 \%$ for patient case-mix.

Although physician effects explained a smaller percentage of the variation seen in the use of extended fractionation, they still accounted for a larger proportion of the variation than did patient case-mix, with $14.4 \%$ versus $7.0 \%$ attributed to physician variation versus patient case-mix for extended fractionation to any site and $20.0 \%$ versus $4.4 \%$ for extended fractionation to bone metastases. Finally, we found that for the use of advanced technologies and for extended fractionation, the random-effects variance was statistically significant in all models, meaning that choice of treatment was influenced by systematic differences in individual radiation oncologist practice patterns rather than by chance. Notably, a later year of diagnosis was associated with less use of extended fractionation but increased 
A

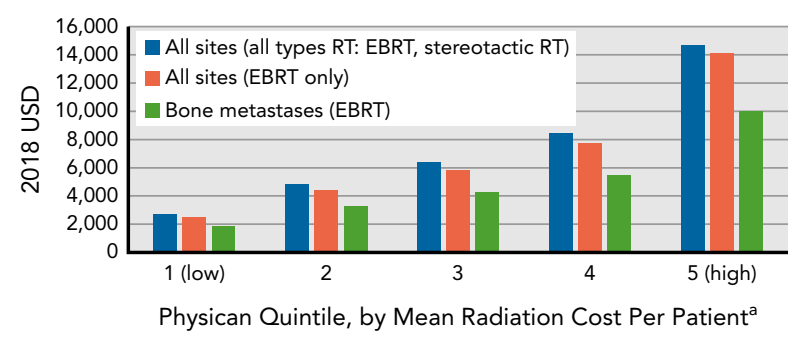

B

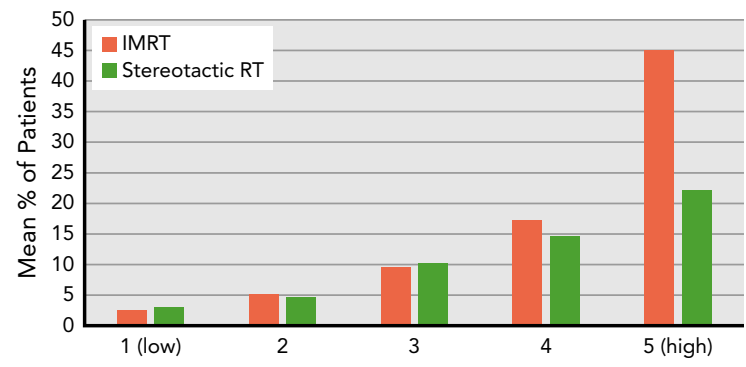

Physican Quintile, by Mean Radiation Cost Per Patient ${ }^{\mathrm{b}}$

\section{C}

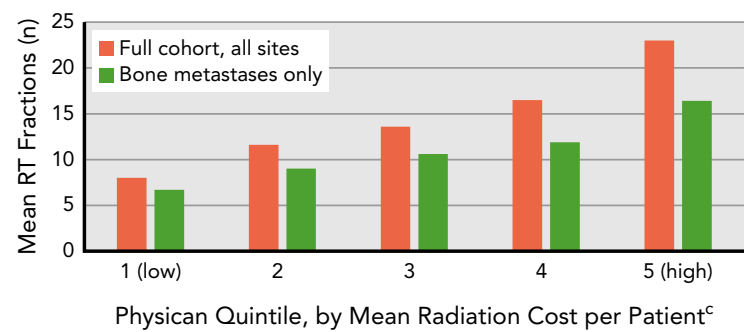

Figure 2. (A) Medicare reimbursement, (B) use of advanced technologies $^{a}$, and $(C)$ number of radiation fractions ${ }^{b}$ by physician cost quintile. For each physician, the mean number of fractions received by patients or the percentage of patients receiving a particular technology was calculated. Descriptive statistics were calculated from these physician-level estimates. Estimates not adjusted by patient case-mix.

Abbreviations: EBRT, external-beam radiation therapy; IMRT, intensitymodulated radiation therapy; RT, radiation therapy.

apercentage of patients receiving IMRT was calculated from among all patients treated with EBRT (IMRT and non-IMRT EBRT); percentage receiving stereotactic RT was calculated from among all patients treated using all types of RT (EBRT or stereotactic RT).

bNumber of radiation fractions was calculated from among all patients treated using EBRT. Patients receiving stereotactic RT were not included because the definition of stereotactic $\mathrm{RT}$ requires $<5$ fractions.

use of advanced technologies. Sensitivity analyses varying the length of the episode to 30 days and including patients who had evidence of multiple courses of radiation occurring within the episode did not substantially affect these results. Additional sensitivity analyses, excluding the $27 \%$ of physicians with only 1 patient in the cohort, also did not substantially affect the results.
Radiation oncologists' years since medical school graduation, medical school location, and sex were available for the $72 \%$ of patients whose claims included an NPI that was recorded in the AMA Physician Masterfile. Among this subgroup of patients, we found that female physicians $(9.6 \%$ vs $11.6 \%$; adjusted odds ratio [aOR], 0.75; 95\% CI, 0.57-0.98; $P=.03$ ), physicians who graduated from medical school after $1990(12.2 \%$ vs 10.5\%; aOR, 1.30; 95\% CI, 1.04-1.62; $P=.03)$, and US medical school graduates $(12.6 \%$ vs $11.0 \%$; aOR, 1.54 ; 95\% CI, 1.15-2.06; $P<.01$ ) were more likely to use IMRT than male physicians, physicians who graduated in 1990 or earlier, and non-US medical school graduates, respectively. Physicians who graduated after 1990 were also more likely to use stereotactic RT than those who graduated in 1990 or earlier ( $11.2 \%$ vs $6.8 \%$; aOR, 1.52; 95\% CI, 1.19-1.94; $P<.01$ ), and female physicians were less likely to use extended fractionation for any site compared with male physicians $(49.3 \%$ vs $54.0 \%$; aOR, 0.83; 95\% CI, 0.72-0.96; $P=.02$ ). However, even when these individual physician characteristics were included in the multilevel model, physician random effects continued to explain a greater proportion of the variation in choice of fractionation and technology use compared with patient-level fixed effects.

After dividing the cohort into those who had survived for $<6$ versus $\geq 6$ months, we found that in both cohorts physician variation continued to explain a larger proportion of the variation in fractionation and use of advanced technologies compared with patient case-mix. In the cohort surviving $<6$ months, physician random effects versus patient case-mix explained $39.4 \%$ versus $14.7 \%$ of IMRT, $45.2 \%$ versus $30 \%$ of stereotactic RT, $13.1 \%$ versus $3.7 \%$ of extended fractionation to any site, and $12.7 \%$ versus $5.1 \%$ of extended fractionation for bone metastases. For the cohort surviving $\geq 6$ months, physician random effects versus patient case-mix explained $28.8 \%$ versus $18.5 \%$ of IMRT, $41.9 \%$ versus $19.1 \%$ of stereotactic RT, $20.0 \%$ versus $10.2 \%$ of extended fractionation to any site, and $29.6 \%$ versus $1.4 \%$ of extended fractionation for bone metastases.

\section{Discussion}

We found that variations in the use of IMRT, stereotactic RT, and extended fractionation among patients with metastatic solid tumors were more strongly associated with differences in radiation oncologists behavior than with patient case-mix. As expected, there was far greater use of high-cost technologies and extended radiation courses among the highest-cost physicians.

We observed that although the use of extended fractionation decreased over time, the use of advanced 


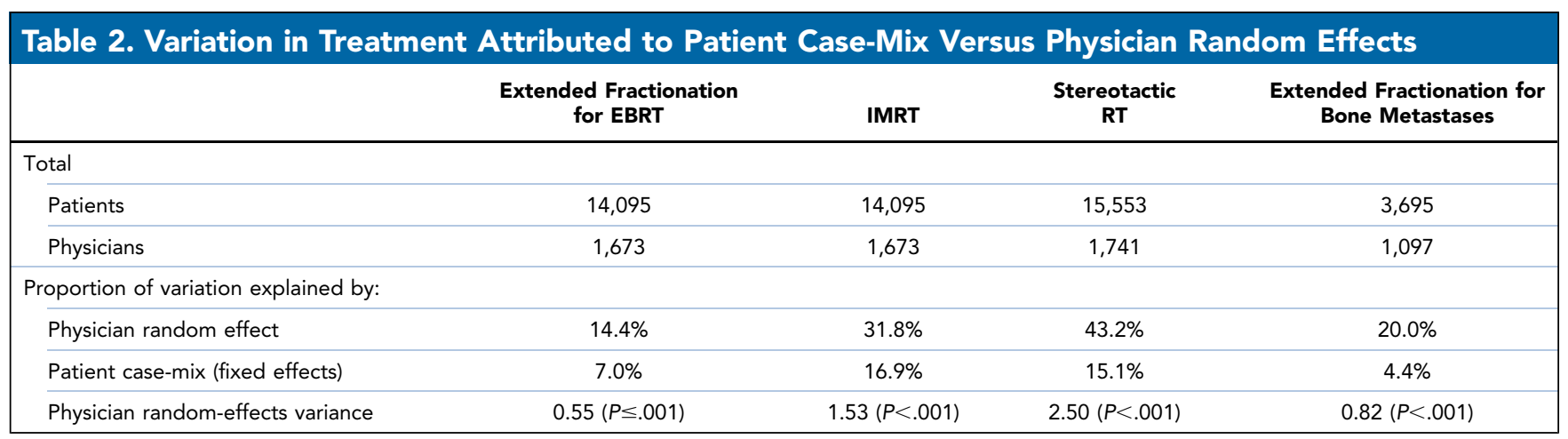

The models include patient-level fixed effects and a random intercept for physicians.

Abbreviations: EBRT, external-beam radiation therapy; IMRT, intensity-modulated radiation therapy; RT, radiation therapy.

technologies increased. This reduction in the use of extended fractionation is consistent with multiple studies showing no significant difference in pain control among patients with uncomplicated bone metastases who receive single versus multifraction treatment ${ }^{10,11,20}$ and subsequent national efforts to reduce the number of radiation fractions used in palliative treatment. ${ }^{11,21,22}$

Nevertheless, we found that the effect of physician variation on care was more pronounced for the adoption of high-cost radiation technologies, such as IMRT and stereotactic RT, than for the number of treatments delivered. This finding suggests that efforts to reduce the costs of palliative RT should not be limited to guidelines on fractionation. Radiation oncologists often decide on the number of treatments delivered based on prior training and experience, whereas new technologies require capital investments and additional effort in training personnel, which may be more variably adopted. Recent studies have also shown a trend toward greater use of advanced RT technologies, such as IMRT and stereotactic radiosurgery, in the last month of life, despite greater costs and scant data showing incremental clinical benefit in this context. ${ }^{23}$ Physician variation may also be a consequence of variability in local healthcare markets and physician compensation models. For example, prior studies have shown differences in the use of IMRT among patients treated in freestanding versus hospital-based radiation facilities, those treated at self-referring versus non-selfreferring practices, and those treated in regions with favorable versus unfavorable IMRT reimbursement policies, suggesting that financial factors may play a role. ${ }^{24-26}$ In our analysis, we likewise observed a greater use of IMRT and extended fractionation at freestanding versus hospital-based facilities. On the other hand, the use of stereotactic RT was more common at hospital-based facilities, likely because of a greater need for specialized personnel and physician involvement.

Finally, patient case-mix variables such as patient age, sex, race, comorbidity score, marital status, region, rural residence, cancer type, and site of metastases influenced the use of radiation technology and extended fractionation. However, these patient factors combined accounted for a smaller portion of variations in the use of high-cost technologies and of extended fractionation compared with radiation oncologists' choices.

There are several limitations to our analysis. First, our data were limited to patients aged $>65$ years living in SEER surveillance areas and enrolled in fee-for-service Medicare; thus these data may not be generalizable to other populations. However, because most patients with cancer are diagnosed at age $>65$ years, and because feefor-service Medicare provides insurance coverage for most Americans aged $>65$ years, our cohort's experience should represent the typical patterns of care for metastatic cancer, and the SEER-Medicare population has previously been shown to be representative of the general population. ${ }^{27,28}$

In addition, SEER-Medicare data lack the clinical details necessary to identify radiation doses, fields, and volumetric and dosimetric data, and it is possible that these factors could affect whether advanced radiation technologies are needed and used. Some patients may be treated at multiple sites, either simultaneously or sequentially. Although we excluded patients who had claims that were consistent with a second course of RT within the 90-day episode of care, we were not able to identify patients who had treatment to more than 1 site during the same radiation course, because only 1 claim is submitted per RT delivery, regardless of the number of sites treated. Moreover, although patient case-mix and treating physician explained approximately half of the observed variation in use of advanced radiation technologies, there remained substantial unexplained 
Table 3. Factors (Fixed Effects) Associated With Radiation Technology and Extended Fractionation

\begin{tabular}{|c|c|c|c|c|c|c|c|c|}
\hline \multirow[b]{2}{*}{ Fixed Effects } & \multicolumn{2}{|c|}{$\begin{array}{c}\text { Extended Fractionation } \\
\text { for EBRT }\end{array}$} & \multicolumn{2}{|l|}{ IMRT } & \multicolumn{2}{|c|}{ Stereotactic RT } & \multicolumn{2}{|c|}{$\begin{array}{l}\text { Extended Fractionation } \\
\text { for Bone Metastases }\end{array}$} \\
\hline & OR $(95 \% \mathrm{Cl})$ & $P$ Value & OR $(95 \% \mathrm{Cl})$ & $P$ Value & OR $(95 \% \mathrm{Cl})$ & $P$ Value & OR $(95 \% \mathrm{Cl})$ & $P$ Value \\
\hline Age group, y & & $<.001$ & & .005 & & .072 & & .255 \\
\hline $66-69$ & Ref & & Ref & & Ref & & Ref & \\
\hline $70-74$ & $1.00(0.92-1.09)$ & & $0.97(0.85-1.11)$ & & $1.16(1.01-1.32)$ & & $1.15(0.96-1.39)$ & \\
\hline $75-79$ & $0.99(0.89-1.09)$ & & $1.03(0.88-1.19)$ & & $1.09(0.93-1.27)$ & & $1.16(0.95-1.42)$ & \\
\hline$\geq 80$ & $0.78(0.71-0.86)$ & & $0.81(0.70-0.94)$ & & $1.20(1.04-1.40)$ & & $1.01(0.83-1.22)$ & \\
\hline Sex & & .031 & & .371 & & .443 & & .376 \\
\hline Female & Ref & & Ref & & Ref & & Ref & \\
\hline Male & $0.92(0.86-0.99)$ & & $1.05(0.94-1.18)$ & & $0.96(0.86-1.07)$ & & $0.93(0.79-1.09)$ & \\
\hline Race & & .372 & & .468 & & .049 & & .494 \\
\hline White & Ref & & Ref & & Ref & & Ref & \\
\hline Black & $0.97(0.85-1.11)$ & & $0.94(0.77-1.15)$ & & $0.75(0.59-0.96)$ & & $1.00(0.75-1.33)$ & \\
\hline Other & $1.12(0.94-1.34)$ & & $0.86(0.65-1.13)$ & & $1.00(0.78-1.28)$ & & $1.22(0.89-1.68)$ & \\
\hline Rural residence & & .958 & & .549 & & .123 & & .112 \\
\hline No & Ref & & Ref & & Ref & & Ref & \\
\hline Yes & $1.00(0.90-1.10)$ & & $0.96(0.84-1.10)$ & & $0.88(0.75-1.03)$ & & $0.86(0.72-1.04)$ & \\
\hline State Medicaid buy-in & & .014 & & .626 & & .019 & & .372 \\
\hline No & Ref & & Ref & & Ref & & Ref & \\
\hline Yes & $0.88(0.79-0.97)$ & & $0.96(0.83-1.12)$ & & $0.82(0.70-0.97)$ & & $0.91(0.73-1.13)$ & \\
\hline College education quintile & & .997 & & .345 & & .823 & & .289 \\
\hline 1 (lowest) & Ref & & Ref & & Ref & & Ref & \\
\hline 2 & $1.00(0.89-1.11)$ & & $0.88(0.75-1.04)$ & & $0.96(0.81-1.13)$ & & $0.99(0.81-1.22)$ & \\
\hline 3 & $1.01(0.90-1.12)$ & & $0.89(0.76-1.04)$ & & $0.99(0.84-1.17)$ & & $1.08(0.87-1.34)$ & \\
\hline 4 & $0.99(0.88-1.11)$ & & $0.92(0.77-1.09)$ & & $0.98(0.82-1.17)$ & & $0.91(0.73-1.14)$ & \\
\hline 5 & $0.98(0.88-1.10)$ & & $1.00(0.83-1.20)$ & & $1.06(0.89-1.27)$ & & $0.86(0.69-1.08)$ & \\
\hline Poverty rate & & .606 & & .146 & & .687 & & .883 \\
\hline Low $(<10 \%)$ & Ref & & Ref & & Ref & & Ref & \\
\hline High ( $\geq 10 \%)$ & $0.98(0.91-1.05)$ & & $0.92(0.81-1.03)$ & & $0.98(0.87-1.10)$ & & $0.99(0.85-1.15)$ & \\
\hline Region & & .004 & & .115 & & .010 & & .013 \\
\hline Northeast & Ref & & Ref & & Ref & & Ref & \\
\hline South & $1.19(1.02-1.40)$ & & $0.91(0.69-1.20)$ & & $0.87(0.65-1.17)$ & & $1.10(0.85-1.44)$ & \\
\hline Midwest & $0.96(0.80-1.16)$ & & $0.99(0.71-1.38)$ & & $0.88(0.64-1.23)$ & & $0.82(0.61-1.10)$ & \\
\hline West & $1.22(1.06-1.41)$ & & $1.18(0.94-1.48)$ & & $1.28(0.98-1.67)$ & & $1.27(1.02-1.58)$ & \\
\hline Marital status & & $<.001$ & & .439 & & .580 & & .001 \\
\hline Married/Partner & Ref & & Ref & & Ref & & Ref & \\
\hline Single & $0.86(0.81-0.93)$ & & $0.96(0.86-1.07)$ & & $1.03(0.93-1.15)$ & & $0.79(0.68-0.91)$ & \\
\hline Modified CCl score & & .027 & & .366 & & .421 & & .383 \\
\hline 0 & Ref & & Ref & & Ref & & Ref & \\
\hline 1 & $1.00(0.93-1.08)$ & & $0.93(0.82-1.05)$ & & $1.07(0.95-1.21)$ & & $0.89(0.75-1.05)$ & \\
\hline$\geq 2$ & $0.89(0.82-0.97)$ & & $1.02(0.90-1.16)$ & & $1.08(0.94-1.24)$ & & $0.98(0.82-1.17)$ & \\
\hline Treatment location & & $<.001$ & & $<.001$ & & $<.001$ & & $<.001$ \\
\hline Freestanding & Ref & & Ref & & Ref & & Ref & \\
\hline Hospital & $0.74(0.67-0.82)$ & & $0.55(0.46-0.65)$ & & $1.66(1.35-2.04)$ & & $0.69(0.58-0.83)$ & \\
\hline
\end{tabular}




\begin{tabular}{|c|c|c|c|c|c|c|c|c|}
\hline \multirow[b]{2}{*}{ Fixed Effects } & \multicolumn{2}{|c|}{$\begin{array}{c}\text { Extended Fractionation } \\
\text { for EBRT }\end{array}$} & \multicolumn{2}{|l|}{ IMRT } & \multicolumn{2}{|c|}{ Stereotactic RT } & \multicolumn{2}{|c|}{$\begin{array}{l}\text { Extended Fractionation } \\
\text { for Bone Metastases }\end{array}$} \\
\hline & OR $(95 \% \mathrm{Cl})$ & $P$ Value & OR $(95 \% \mathrm{Cl})$ & $P$ Value & OR $(95 \% \mathrm{Cl})$ & $P$ Value & OR $(95 \% \mathrm{Cl})$ & $P$ Value \\
\hline Cancer type & & $<.001$ & & $<.001$ & & .001 & & .176 \\
\hline Lung & Ref & & Ref & & Ref & & Ref & \\
\hline Breast & $1.45(1.25-1.68)$ & & $0.93(0.73-1.19)$ & & $0.80(0.62-1.03)$ & & $1.22(0.96-1.56)$ & \\
\hline Prostate & $1.18(1.03-1.34)$ & & $2.21(1.81-2.68)$ & & $0.59(0.44-0.80)$ & & $1.18(0.97-1.44)$ & \\
\hline Colorectal & $2.46(2.05-2.94)$ & & $1.74(1.42-2.13)$ & & $1.25(0.97-1.62)$ & & $0.97(0.53-1.75)$ & \\
\hline \multicolumn{9}{|c|}{ Metastases at diagnosis } \\
\hline Bone (yes vs no) & $0.52(0.49-0.56)$ & $<.001$ & $0.33(0.29-0.38)$ & $<.001$ & $0.49(0.44-0.56)$ & $<.001$ & Ref & \\
\hline Brain (yes vs no) & $0.68(0.62-0.74)$ & $<.001$ & $0.27(0.22-0.32)$ & $<.001$ & $3.37(2.94-3.86)$ & $<.001$ & $1.62(1.29-2.03)$ & $<.001$ \\
\hline Liver (yes vs no) & $0.61(0.56-0.67)$ & $<.001$ & $0.61(0.53-0.71)$ & $<.001$ & $0.72(0.62-0.83)$ & $<.001$ & $0.64(0.53-0.78)$ & $<.001$ \\
\hline Lung (yes vs no) & $0.95(0.87-1.02)$ & .161 & $0.94(0.84-1.05)$ & .274 & $0.99(0.88-1.12)$ & .907 & $0.92(0.77-1.10)$ & .332 \\
\hline Year of diagnosis & & $<.001$ & & $<.001$ & & $<.001$ & & $<.001$ \\
\hline 2010 & Ref & & Ref & & Ref & & Ref & \\
\hline 2011 & $1.00(0.90-1.12)$ & & $1.40(1.17-1.67)$ & & $1.30(1.08-1.56)$ & & $1.01(0.80-1.27)$ & \\
\hline 2012 & $0.86(0.76-0.96)$ & & $1.26(1.03-1.54)$ & & $1.49(1.23-1.80)$ & & $0.93(0.74-1.16)$ & \\
\hline 2013 & $0.77(0.68-0.86)$ & & $1.44(1.19-1.75)$ & & $1.69(1.39-2.07)$ & & $0.69(0.54-0.87)$ & \\
\hline 2014 & $0.78(0.69-0.88)$ & & $1.87(1.54-2.27)$ & & $1.79(1.48-2.16)$ & & $0.67(0.54-0.85)$ & \\
\hline 2015 & $0.74(0.66-0.84)$ & & $2.36(1.94-2.87)$ & & $2.10(1.73-2.57)$ & & $0.67(0.53-0.85)$ & \\
\hline
\end{tabular}

The models include patient-level fixed effects and a random intercept for physicians.

Abbreviations: CCl, Charlson comorbidity index; EBRT, external-beam radiation therapy; IMRT, intensity-modulated radiation therapy; OR, odds ratio; RT, radiation therapy. apercent of persons aged $\geq 25$ years with at least 4 years of college education in the patient's census tract.

variation in treatment, which could result not only from random variation in treatment but also from unobserved characteristics, such as the local healthcare market environment or other unobserved patient and provider characteristics.

Furthermore, it is possible that differences in the costs of radiation could be offset by a reduction in adverse effects or improvement in outcomes. For example, use of IMRT has been associated with reduction of pneumonitis in patients with lung cancer and salivary function in those with head and neck cancer treated with curative intent, ${ }^{29,30}$ although there is no strong evidence of similar benefit in patients receiving palliative radiation. Cost-effectiveness studies of palliative radiation have shown greater cost-effectiveness with single-fraction versus multifraction radiation, ${ }^{31}$ although there are clinical contexts in which stereotactic RT may be cost-effective compared with conventional radiation. ${ }^{32,33}$

An additional limitation of our analysis is that outcomes related to palliation such as pain control are not available in the claims data, and toxicities from radiation such as esophagitis may not be reliably captured in claims data unless they are severe enough to require medical treatment. Although multiple studies of radiation for bone metastases have not found extended fractionation to be beneficial, the introduction of new systemic therapies, including targeted therapies and immunotherapy that are associated with improvements in prognosis, could shift this balance, particularly in patients with oligometastatic disease. Finally, although reducing variation in use of high-cost technologies can help reduce the costs of palliative RT, these costs constitute a small proportion of overall cancer and end-oflife spending. ${ }^{34,35}$

\section{Conclusions}

Findings from our study support efforts to examine radiation oncologists' discretionary use of high-cost technologies. Although we have shown that variation in care is associated with differences in mean costs of palliative RT, by physician, additional work is needed to determine whether this variation in care affects patient outcomes. There are certainly situations in which use of high-cost technologies may be justified, such as for reirradiation and treatment of radioresistant tumors. Advanced and often high-cost technologies are attractive for their ability to improve accuracy and reduce toxicity from RT, but their impact on palliative endpoints has not been as well established as it has been for curative-intent RT. Furthermore, as alternative payment models for radiation oncology are 
implemented, including proposed episode-based payment models, we will be able to better examine the extent to which physician variation is a consequence of financial incentives versus other factors affecting physician practice, such as availability of resources and local referral and practice patterns.

\section{Acknowledgments}

Dr. Ya-Chen Tina Shih is supported by NCI R01 CA207216. Dr. Sharon Giordano is supported by CPRIT RP160674, Komen SAC150061, and NCI P30 CA016672. This study used the linked SEER-Medicare database. The interpretation and reporting of these data are the sole responsibility of the authors. The authors acknowledge the efforts of the Applied Research Program at NCI; the Office of Research, Development, and Information, Centers for Medicare \& Medicaid Services; Information Management Services, Inc.; and the
SEER program tumor registries in the creation of the SEERMedicare database.

Submitted January 6, 2020; accepted for publication August 3, 2020 Published online February 12, 2021.

Author contributions: Study concept and design: Chen, Niu, Cronin, Shih, Schrag. Data acquisition, analysis, and interpretation: All authors. Manuscript preparation: Chen, Niu, Cronin, Schrag. Critical revision: All authors

Disclosures: Dr. Shih has disclosed that she has received consulting fees from Pfizer Inc. and is a scientific advisor for AstraZeneca. Dr. Schrag has disclosed that she has received funding from JAMA for editorial services, funding from Pfizer, and institutional research funding from GRAIL and AACR. The remaining authors have disclosed that they have not received any financial consideration from any person or organization to support the preparation, analysis, results, or discussion of this article.

Funding: This study was supported by grants from the Gloria Spivak Fund and the American Society for Radiation Oncology.

Correspondence: Aileen B. Chen, MD, MPP, The University of Texas MD Anderson Cancer Center, 1515 Holcombe Boulevard, Unit 97, Houston, TX 77030. Email: achen6@mdanderson.org

\section{References}

1. Centers for Medicare \& Medicaid Services. Radiation oncology model. Accessed April 28, 2020. Available at: https://innovation.cms.gov/innovation-models/radiation-oncology-model

2. Centers for Medicare \& Medicaid Services. Oncology care model. Accessed April 28, 2020. Available at: https://innovation.cms.gov/innovation-models/oncology-care

3. Reschovsky JD, Hadley J, Romano PS. Geographic variation in feefor-service Medicare beneficiaries' medical costs is largely explained by disease burden. Med Care Res Rev 2013;70:542-563.

4. Gripp S, Mjartan S, Boelke E, et al. Palliative radiotherapy tailored to life expectancy in end-stage cancer patients: reality or myth? Cancer 2010; 116:3251-3256

5. Gomez DR, Tang C, Zhang J, et al. Local consolidative therapy vs. maintenance therapy or observation for patients with oligometastatic non-small-cell lung cancer: long-term results of a multiinstitutional, phase II, randomized study. J Clin Oncol 2019;37: 1558-1565.

6. Palma DA, Olson $\mathrm{R}$, Harrow $\mathrm{S}$, et al. Stereotactic ablative radiotherapy for the comprehensive treatment of 4-10 oligometastatic tumors (SABR-COMET-10): study protocol for a randomized phase III trial. BMC Cancer 2019;19:816.

7. Sahgal A, Aoyama H, Kocher M, et al. Phase 3 trials of stereotactic radiosurgery with or without whole-brain radiation therapy for 1 to 4 brain metastases: individual patient data meta-analysis. Int J Radiat Oncol Biol Phys 2015;91:710-717.

8. Parikh RB, Cronin AM, Kozono DE, et al. Definitive primary therapy in patients presenting with oligometastatic non-small cell lung cancer. Int J Radiat Oncol Biol Phys 2014;89:880-887.

9. Dans M, Kutner JS, Agarwal R, et al. NCCN Clinical Practice Guidelines in Oncology: Palliative Care. Version 1.2021. Accessed December 2, 2020. To view the most recent version, visit NCCN.org

10. Chow E, Harris K, Fan G, et al. Palliative radiotherapy trials for bone metastases: a systematic review. J Clin Oncol 2007;25:1423-1436.

11. Lutz S, Berk L, Chang E, et al. Palliative radiotherapy for bone metastases: an ASTRO evidence-based guideline. Int J Radiat Oncol Biol Phys 2011; 79:965-976.

12. Radiation Oncology Alternative Payment Model (RO-APM) Accessed August 11, 2020. Available at: https://www.astro.org/ uploadedFiles/_MAIN_SITE/Daily_Practice/Medicare_Payment_Initiatives/Alternative Payment_Model_Program/Content_Pieces/ ROAPM_Description.pdf

13. U.S. Department of Health and Human Services. Report to Congress: episodic alternative payment model for radiation therapy services. Accessed August 11, 2020. Available at: https://innovation.cms.gov/ Files/reports/radiationtherapy-apm-rtc.pdf

14. Warren JL, Yabroff KR, Meekins A, et al. Evaluation of trends in the cost of initial cancer treatment. J Natl Cancer Inst 2008:100:888-897.
15. Brown ML, Riley GF, Schussler N, et al. Estimating health care costs related to cancer treatment from SEER-Medicare data. Med Care 2002 40(8 Suppl):IV-104-117

16. Deyo RA, Cherkin DC, Ciol MA. Adapting a clinical comorbidity index for use with ICD-9-CM administrative databases. J Clin Epidemiol 1992;45:613-619.

17. Klabunde CN, Potosky AL, Legler JM, et al. Development of a comorbidity index using physician claims data. J Clin Epidemiol 2000;53:1258-1267.

18. Charlson ME, Pompei $\mathrm{P}$, Ales $\mathrm{KL}$, et al. A new method of classifying prognostic comorbidity in longitudinal studies: development and validation. J Chronic Dis 1987;40:373-383.

19. Austin PC, Merlo J. Intermediate and advanced topics in multilevel logistic regression analysis. Stat Med 2017;36:3257-3277

20. Sze WM, Shelley M, Held I, et al. Palliation of metastatic bone pain: single fraction versus multifraction radiotherapy-a systematic review of the randomised trials. Cochrane Database Syst Rev 2004:CD004721.

21. Lutz S, Balboni T, Jones J, et al. Palliative radiation therapy for bone metastases: update of an ASTRO evidence-based guideline. Pract Radiat Oncol 2017;7:4-12.

22. Choosing Wisely. ASTRO - extended fractionation schemes for palliation of bone metastases. Accessed August 11, 2020. Available at: http://www. choosingwisely.org/clinician-lists/american-society-radiation-oncologyextended-fractionation-schemes-for-palliation-of-bone-metastases/

23. Guadagnolo BA, Liao KP, Giordano SH, et al. Increasing use of advanced radiation therapy technologies in the last 30 days of life among patients dying as a result of cancer in the United States. J Oncol Pract 2014:10:e269-276.

24. Chen AB, Li L, Cronin A, et al. Comparative effectiveness of intensity-modulated versus $3 D$ conformal radiation therapy among Medicare patients with stage III lung cancer. J Thorac Oncol 2014;9: 1788-1795.

25. Mitchell JM. Urologists' use of intensity-modulated radiation therapy for prostate cancer. N Engl J Med 2013;369:1629-1637.

26. Smith BD, Pan IW, Shih YC, et al. Adoption of intensity-modulated radiation therapy for breast cancer in the United States. J Natl Cancer Inst 2011;103:798-809.

27. Nattinger AB, McAuliffe TL, Schapira MM. Generalizability of the surveillance, epidemiology, and end results registry population: factors relevant to epidemiologic and health care research. J Clin Epidemiol 1997;50:939-945

28. American Cancer Society. Key statistics for lung cancer. Accessed August 11, 2020. Available at: https://www.cancer.org/cancer/lung-cancer/ about/key-statistics.html

29. Chun SG, Hu C, Choy $\mathrm{H}$, et al. Impact of intensity-modulated radiation therapy technique for locally advanced non-small-cell lung cancer: a secondary analysis of the NRG Oncology RTOG 0617 randomized clinical trial. J Clin Oncol 2017;35:56-62.

30. Nutting CM, Morden JP, Harrington KJ, et al. Parotid-sparing intensity modulated versus conventional radiotherapy in head and neck cancer 
(PARSPORT): a phase 3 multicentre randomised controlled trial. Lancet Oncol 2011;12:127-136.

31. Konski A. Radiotherapy is a cost-effective palliative treatment for patients with bone metastasis from prostate cancer. Int J Radiat Oncol Biol Phys 2004;60:1373-1378.

32. Lester-Coll NH, Dosoretz AP, Magnuson WJ, et al. Cost-effectiveness of stereotactic radiosurgery versus whole-brain radiation therapy for up to 10 brain metastases. J Neurosurg 2016;125(Suppl 1):18-25

33. Kim H, Rajagopalan MS, Beriwal S, et al. Cost-effectiveness analysis of single fraction of stereotactic body radiation therapy compared with single fraction of external beam radiation therapy for palliation of vertebral bone metastases. Int J Radiat Oncol Biol Phys 2015;91:556-563.

34. Fitch K, Pelizzari PM, Pyenson B. Cost drivers of cancer care: a retrospective analysis of Medicare and commercially insured population claim data 2004-2014. Accessed August 11, 2020. Available at: https://communityoncology.org/pdfs/Trends-in-Cancer-Costs-White-Paper-FINAL20160403.pdf

35. Brooks GA, Li L, Uno H, et al. Acute hospital care is the chief driver of regional spending variation in Medicare patients with advanced cancer. Health Aff (Millwood) 2014;33:1793-1800.

\section{See JNCCN.org for supplemental online content.}


Supplemental online content for:

\section{Variation in Use of High-Cost Technologies for Palliative Radiation Therapy by Radiation Oncologists}

Aileen B. Chen, MD, MPP; Jiangong Niu, PhD; Angel M. Cronin, MS; Ya-Chen Tina Shih, PhD;

Sharon Giordano, MD, MPH; and Deborah Schrag, MD, MPH

J Natl Compr Canc Netw 2021;19(4):421-431

eFigure 1: Construction of Patient Cohort 

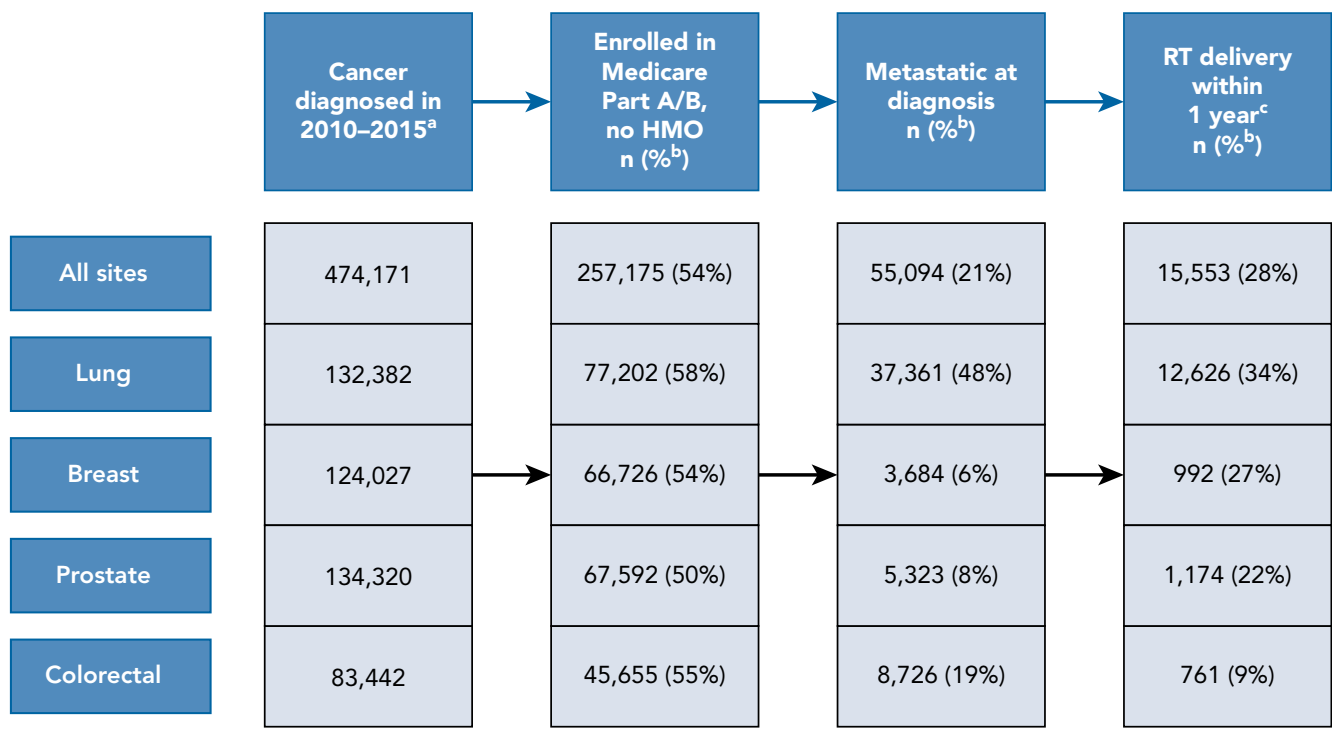

eFigure 1. Construction of Patient Cohort.

Abbreviation: RT, radiation therapy.

aPatients aged $>65$ years, no prior cancers, not enrolled because of end-stage renal disease or disability, not diagnosed by autopsy/death certificate.

bRemaining from prior step.

Inclusion criteria: RT planning code was no more than 28 days before and no more than 3 days after the first RT delivery code. RT assignable to specific physician, with minimum 90 days of claims data available. Note: $>99 \%$ met all inclusion criteria for each disease site. 